\title{
DeVelopment of a Noninvasive Diabetes SCREENING DeVice USING THE RATIO OF FLUORESCEnce TO RAYLEIGH SCATTERED LIGHT
}

\author{
Nai-Teng Yu, ${ }^{\dagger}$ Brian S. Krantz, ${ }^{\ddagger}$ Jonathan A. Eppstein, ${ }^{\ddagger}$ Keith D. Ignotz,${ }^{\ddagger}$ \\ Mark A. Samuels, ${ }^{*}$ James R. Long, ${ }^{*}$ and John F. Price* \\ ${ }^{\dagger}$ Hong Kong University of Science and Technology, Department of Chemistry, Clear Water Bay, \\ Kowloon, Hong Kong; ${ }^{\ddagger}$ SpectRx, Inc., Research Department, Norcross, Georgia 30071; ${ }^{*}$ Boehringer \\ Mannheim Corporation, Research and Development, Indianapolis, Indiana 46250 \\ (Paper JBO/LSF-02 received Mar. 8, 1996; revised manuscript received May 2, 1996; accepted for publication May 9, 1996)
}

\begin{abstract}
We have developed a new lens measurement system (LMS) that simultaneously measures the intensities of fluorescence and Rayleigh components at various distances into the lens along the optical axis. The noninvasive measurement is performed through an undilated pupil, and with the assistance of a pupil tracking system that facilitates maintaining the $x$ and $y$ positions of the sample volume (ca. $300 \mu \mathrm{m}$ in length, $600 \mu \mathrm{m}$ high, and $80 \mu \mathrm{m}$ wide) to within $\pm 100 \mu \mathrm{m}$ of any programmed "lock" position. The intensity of the Rayleigh component that is used to normalize the measured fluorescent signal serves to correct the attenuation effects due to absorption and lens light scatter (Mie-Tyndall type). This report, resulting from a SpectRx Site L clinical study using a refined instrumentation (SpecRx Scan V), presents analysis of fluorescence and Rayleigh data from the lenses of 923 controls and 239 diabetic subjects (45 Type I and 194 Type II) ranging from 23 to 75 years old. Fluorescence and Rayleigh data have been obtained via confocal mode from various locations nominally along the lens optical axis (the true trajectory of a sample volume could be shown in a figure) for controls and diabetics, at different ages, using three pairs of excitation and collection wavelengths: $364 / 495$ $\mathrm{nm}, 434 / 495 \mathrm{~nm}$, and 485/515 nm. For control subjects, there exists a strong, almost linear relationship between age and fluorescence, while diabetic subjects tend to deviate from this age-fluorescence relationship. Our data show that the lenses of diabetic patients are subject to an accelerated aging process, presumably due to an elevated level of brown and fluorescent protein adducts and crosslinks from nonenzymatic glycosylation (Maillard-Amadori reactions). We have also shown that by using the measured Rayleigh profiles to normalize the measured fluorescence, most of the absorption effects are removed and therefore the separation between the fluorescence of diabetics and controls is greatly improved. Thus, the device for measuring fluorescence/Rayleigh ratios can be used to noninvasively screen populations for possible undiagnosed diabetes. $\odot 1996$ Society of Photo-Optical Instrumentation Engineers.
\end{abstract}

Keywords light scatter and fluorescence; Rayleigh scattered light; diabetes; lens measurement system.

\section{INTRODUCTION}

The fluorescence of human lenses has been the subject of numerous investigations regarding the mechanisms of its production, ${ }^{1-6}$ the chemical structure of the fluorophores, ${ }^{7-11}$ and their possible involvement in cataractogenesis. ${ }^{12,13}$ The infant lens contains a yellow pigment, known to be 3-hydroxyL-kynurenine- $O$ - $\beta$-glucoside, ${ }^{1}$ which exhibits a characteristic fluorescence at ca. $440 \mathrm{~nm}$ when excited near $350 \mathrm{~nm}$ (its absorption maximum). ${ }^{14}$ This fluorescence intensity of the infant lens decreases rapidly as the excitation wavelength increases from 350 to ca. $480 \mathrm{~nm}$, above which fluorescence vanishes completely. ${ }^{15}$ Fixing the excitation wavelength at, say, $488 \mathrm{~nm}$, one can observe the appearance of a new fluorescence (emission maximum at ca. 538 $\mathrm{nm}){ }^{15}$ its intensity increases with age, indicating the age-related production of a new fluorophore that is definitely not 3-hydroxy-L-kynurenine-O$\beta$-glucoside. ${ }^{2}$ However, based on evidence from fluorescence distribution profiles, $\mathrm{Yu}$, Barron and Kuck $^{2}$ suggested that this new fluorophore may be a derivative of 3-hydroxy-L-kynurenine-O- $\beta$ glucoside (likely protein bound). Additional fluorophores produced in the older human lens can be detected by excitation at even longer wavelengths $(500-700 \mathrm{~nm}) .{ }^{16}$ The exact nature of these fluorophores is unknown at present.

Lens crystallins, being long-lived protein molecules with little turnover, are particularly susceptible to nonenzymatic glycosylation with glucose,

1083-3668/96/\$6.00 (C) 1996 SPIE 
leading to the subsequent formation of brown and fluorescence adducts and crosslinks (via MaillardAmadori reactions). ${ }^{17-20}$ The fluorescence of these adducts and crosslinks will contribute to the total measured fluorescent signal. The formation of these adducts and crosslinks should be elevated in diabetes. Thus, the lens of a diabetic patient is expected to exhibit more fluorescence, which may be viewed as an indicator for an accelerated aging.

Conventional in vivo measurements of lens fluorescence do not correct the measured fluorescent signal for changes in lens absorption and scatter. This can have a marked effect on the measurements because increased lens absorption and scatter will attenuate both the incident excitation and the emitted fluorescence, resulting in an apparent diminution of the actual lens fluorescence. Preliminary studies at the Joselin Diabetes Center ${ }^{21}$ revealed that if the fluorescence data are corrected by using the intensity of the Rayleigh component for normalization, the separation between the two populations (diabetics vs. nondiabetics) shows great improvement.

This paper reports on our continued efforts in developing a clinically oriented lens measurement system (LMS), and its use in the measurements of lens fluorescence and Rayleigh profiles along the optical axis from a larger population (total no. of subjects $=1,162)$. We demonstrated clearly that Rayleigh-normalized fluorescence provides more sensitive discrimination between diabetic and nondiabetic lenses than the conventional lens fluorescence measurements. Thus, it appears that this device for measuring fluorescence and Rayleigh ratios can be used to noninvasively screen populations for detecting, in particular, undiagnosed Type II diabetic subjects. In the case of Type I diabetic subjects, the onset is typically very rapid, providing essentially a step function in the loss of control of glucose levels; these individuals can be diagnosed by the conventional methods available today. On the other hand, the Type II diabetic subjects, which studies have shown have frequently been undiagnosed for 8 to 12 years prior to the initial diagnosis, would be expected to show clear signs of accelerated lens aging, and thus would be detectable by the LMS.

\section{MATERIAlS AND METHODS}

\subsection{HUMAN SUBJECTS}

Measurements were made from the clear lenses of 923 controls $\left(N_{c}\right), 45$ Type I diabetics $\left(N_{d 1}\right)$, and 194 Type II diabetics $\left(N_{d 2}\right)$. The subjects with cataracts were screened out of the study. The Type I and Type II diabetes patients were merged to yield a total number of diabetes patients, $N_{d}$, of 239 . The inclusion of the 45 Type I diabetics in the calculation of the "average" wave forms has almost no effect on the "average" wave form because there are so few compared with Type IIs. The age distributions of the two populations are shown in Figure
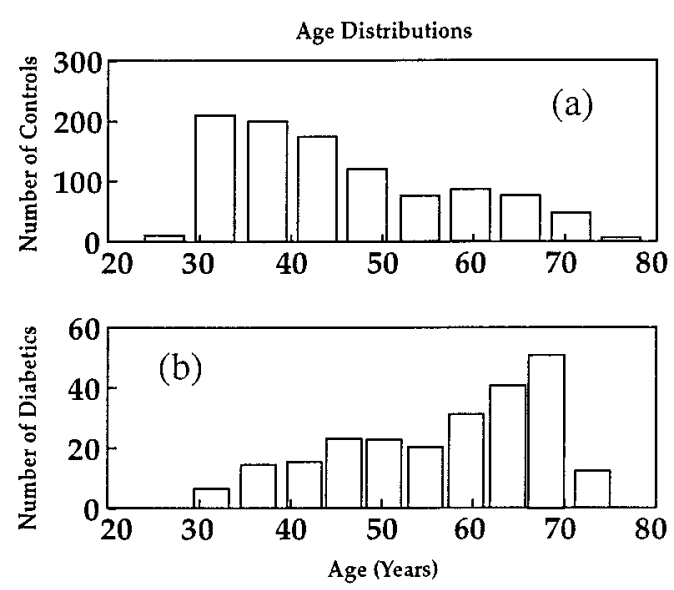

Fig. 1 The age distributions of (a) controls and (b) diabetic subjects.

1(a) and 1(b). Although the two populations have obvious differences in their age distributions, there are enough samples in each age group to eliminate artifactual results caused by age.

\subsection{CLINICAL LENS MEASUREMENT PROTOCOLS}

The lens measurement system employed for the clinical study was a Scan V model developed by SpectRx, Inc. (Norcross, Georgia). The schematic for the LMS is shown in Figure 2. A fiber-coupled $300-\mathrm{W}$ xenon lamp is the excitation source. The device simultaneously measures the intensities of fluorescence and wavelength-unshifted component (loosely called Rayleigh) at various distances into the lens along the optical axis (defined as the $z$ axis in our system) of the human lens in situ. This was accomplished by translating the sample volume of

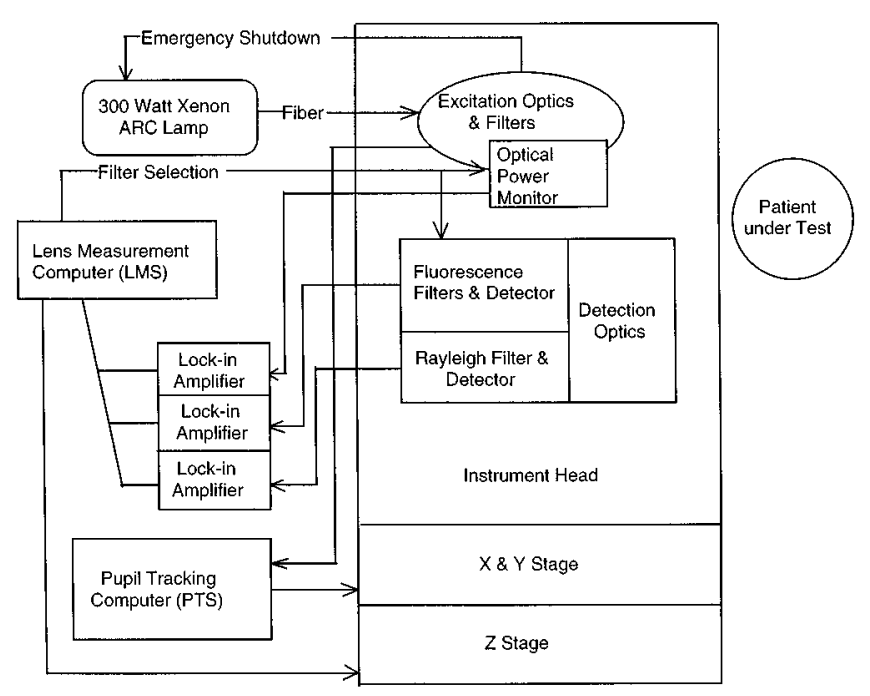

Fig. 2 The schematic of the in vivo lens measurement system (LMS). 
the confocal instrumentation along this $z$ axis. These studies relied on the automatic $x-y-z$ alignment system to preserve the $x$ and $y$ orientation of the lens relative to the sample volume as it was scanned.

A pupil tracking system (PTS) has been incorporated into the Scan V LMS. An infrared CCD camera images the iris and pupil using an optical system concentric with the excitation beam. The CCD image is digitized and the circular pupil is located using a signal-processing algorithm. The position data derived from the digitized CCD image are fed into a two-axis servomotor positioning system. The system facilitates maintaining the $x$ and $y$ positions of the sample volume to within $\pm 100 \mu \mathrm{m}$ of any programmed "lock" position. The optics of LMS defines a sample volume approximately $300 \mu \mathrm{m}$ in length, $600 \mu \mathrm{m}$ high, and $80 \mu \mathrm{m}$ wide. Separate filter wheels for each of the LMS's three optical channels (excitation, collection channel 1, and collection channel 2) allow for 72 possible excitation and collection wavelength combinations from a single broad-spectrum source. The filters were purchased from Omega Optical Inc. (Brattleboro, Vermont) and are of Omega's "DF" series.

The specifications on the filters were important in that they were designed to allow very pure measurement of the fluorescence without any contribution from backscattered light. This alone distinguishes the LMS from earlier efforts to measure lens autofluorescence with the Coherent Fluorotron Master, where the filter leakage of the backscattered light can clearly be seen in the scans through the cornea and lens. In the LMS, all of the filters used in both the excitation channel and the fluorescence receiving channel have an out-of-band rejection greater than optical density (OD) 5. That is, less than $1 / 100,000$ th of the light falling outside the filter's passband will come through. Furthermore, the individual filters were oriented to minimize any beam steering effects of the wedge which may have been present in them. This was to ensure that the confocal spot remained in the same location relative to the instrument regardless of which filters were in place. In addition, a sensor in the excitation channel provides an instantaneous monitor of the optical power delivered to the eye, which can be used to normalize fluctuations in the source output, and provides a failsafe mechanism that shuts the lamp off if the power levels ever exceed a predetermined threshold.

\subsubsection{Preprocessing}

The data from each clinical measurement were stored on a separate computer floppy diskette with binary files containing all of the measurement data and text files that had patient information and hardware and software configuration of the LMS for that measurement. A library of functions was developed to preprocess the data into a form that facilitates the development of the relationship among fluorescence, scattering, and absorption. This process can be divided into three categories: smoothing, normalization, and alignment.

\section{Smoothing}

The signal-to-noise ratio (SNR) of the LMS is quite high (ca. $100 \mathrm{~dB}$ ). However, there are some highfrequency variations. This noise is averaged out by a Gaussian filter that is convolved with each scan waveform. The filter has a full-width at halfmaximum (FWHM) of $150 \mathrm{~nm}$.

\section{Normalization}

Power. The intensities recorded by the LMS are dependent on the lamp power. Therefore, the results must be power normalized to be able to compare data taken at different times. With the present system, there are two different methods one can use to power normalize the data: calibration target peaks, and instantaneous power sensor data.

Calibration target peaks. Before each measurement, a fluorescent calibration target is placed in front of the LMS, and it is scanned with every excitation wavelength used in the subject cycle. The peaks of the fluorescence wave forms are directly proportional to lamp intensity. Therefore, there is one calibration constant for every excitation wavelength.

Power sensor data. As mentioned earlier, a power sensor allows for the instantaneous collection of excitation intensity at each measurement point. This results in a calibration constant for every measurement point.

There are advantages and disadvantages with every normalization method. The key differences are that the power sensor calibration can normalize fluctuations in lamp power during a measurement. Also, power measurements using this sensor can be less noisy than the fluorescence of the calibration target. On the other hand, the calibration target method provides normalization for the entire system while the power sensor only calibrates the lamp and excitation channel. It has not been determined which method, if any, is superior. At present, the dual methods provide redundancy in case of a hardware failure in either system.

Lens width. Studies have shown that lens width increases with age. If we are trying to compare fluorescence and scattering intensities at various distances into the lens, it might be valid to compare an old lens that is $5.5 \mathrm{~mm}$ with a young lens of $4 \mathrm{~mm}$. So, we need to develop a method of lens width normalization. This is accomplished by determining the front and rear of the lens, and then applying a mathematical spline operation to restructure the scan wave forms into a set number of points. The result is a wave form in which each point is a certain percentage through the lens, instead of a certain absolute distance. 


\section{Alignment}

For any type of intersubject comparisons to be meaningful, there must be a fixed method of aligning the wave forms, or scans. This is done by aligning the front edge using the first point where the value exceeds $10 \%$ of the front cortical peak in each scan. We have chosen this method because it limits the effect of absorption on the alignment position.

\subsubsection{Data Processing}

In order to provide better insight into the age contributions in the data, averaging was performed. For each excitation and collection, the wave forms (profiles) were put into "age bins" that spanned 2 years and then averaged. This resulted in 53 average wave forms (profiles) for the controls (23 to 75year-old bins; 10 to 20 wave forms per bin) and 47 average wave forms (29 to 75-year-old bins; 2 to 6 wave forms per bin) for the diabetic subjects.

\section{RESULTS}

We present here the results from the SpectRx Site L clinical study. The data are power normalized using the calibration target and are not normalized for lens width.

\subsection{RELATIVE FLUORESCENCE MEASUREMENTS}

We have obtained relative fluorescence data using three pairs of excitation and collection wavelengths: $434 / 495 \mathrm{~nm}, 485 / 515 \mathrm{~nm}$, and 364/495 nm. The results are a series of profiles (or wave forms) along the optical axis (expressed as displacement in millimeters) for different ages, as shown in Figure 3(a) (controls) and Figure 3(b) (diabetic subjects) with excitation and collection=434/495 nm. Some linear (regression) correlation coefficients, $r$, between fluorescence and age are displayed in Table 1 for controls (a) and diabetic subjects (b). Table 1(c) shows the $p$-value of a linear regression between fluorescence and age (or significant $F$ of the regression) for the 434-nm excitation only. Figure 4(a) to 4(d) show the data extracted from Figure 3(a) to 3(b): the plots of controls and diabetic subjects' fluorescence versus age are shown at 1.75, 2.25, 2.75, and $3.25 \mathrm{~mm}$ into the lens, using excitation and collection at $434 / 495 \mathrm{~nm}$.

\subsection{RELATIVE RAYLEIGH MEASUREMENTS}

The relative intensities of Rayleigh components (at $434 \mathrm{~nm}$ ) along the optical axis (expressed as displacement in millimeters) for different ages are shown in Figure 5(a) (controls) and Figure 5(b) (diabetic subjects). For all ages, the Rayleigh profile exhibits maximum intensities at the anterior and posterior lens capsule interfaces due to the specular component (reflected light) present at these locations which cannot be resolved from the Rayleigh.

\subsection{RAYLEIGH NORMALIZED FLUORESCENCE (FLUORESCENCE/RAYLEIGH RATIO)}

The results of relative fluorescence [Figure 3(a) to 3(b)] normalized by relative Rayleigh [Figure 4(a) to 4(b)] are shown in Figure 6(a) to 6(b). For older control lenses (e.g., 70 years old), there are three maxima (two at the anterior and posterior cortex, and the third in the nuclear region), which is in agreement with the fluorescence profiles obtained from lens slices using similar excitation wavelengths (at $441.6 \mathrm{~nm}){ }^{3}$ The profiles of young control lenses with a maximum near the nucleus center are also in good agreement with the previous results. ${ }^{3}$ Figure $7(\mathrm{a})$ to $7(\mathrm{~d})$ show the data extracted from Figure 6(a) and 6(b): the plots are for Rayleigh normalized fluorescence (for controls and diabetics) versus age at $1.75,2.5,3.25$, and $4 \mathrm{~mm}$ into the lens, using excitation and collection at $434 / 495 \mathrm{~nm}$.

\section{DISCUSSION}

From Figure 3 and Table 1, it is clear that for control subjects, there exists a strong, almost linear relationship between age and fluorescence. However, individuals with diabetes tend to deviate from the normal age-fluorescence relationship. We hypothesize two different explanations for the phenomena.
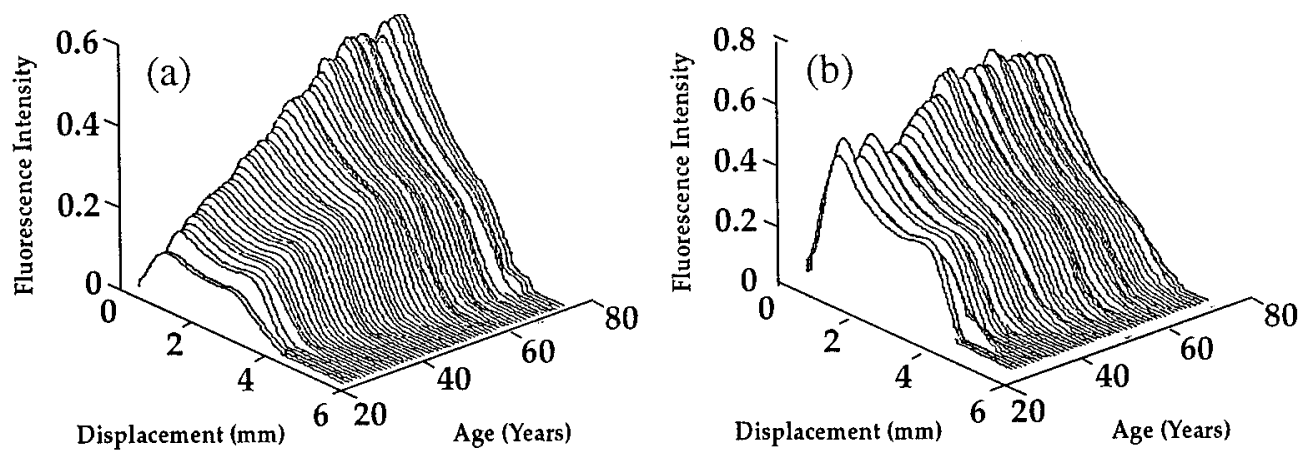

Fig. 3 Relative fluorescence profiles (or wave forms) for different ages along the optical axis of the lens in (a) controls and (b) diabetic subjects. The excitation and collection wavelength $=434 / 495 \mathrm{~nm}$. 
Table 1 (a) Correlation coefficients between flourescence and age of controls. (b) Correlation coefficients between flourescence and age of diabetic subjects. (c) Regression p-values for regressions between age and flourescence resulting from $434 \mathrm{~nm}$ excitation.

(a)

$\begin{array}{lllllllllll}\text { Displacement }(\mathrm{mm}) & 0.25 & 0.75 & 1.25 & 1.75 & 2.25 & 2.75 & 3.25 & 3.75 & 4.25 & 4.75 \\ 485 \mathrm{~nm} \text { Excitation } & 0.953 & 0.969 & 0.993 & 0.995 & 0.992 & 0.980 & 0.954 & 0.946 & 0.910 & 0.786 \\ 434 \mathrm{~nm} \text { Excitation } & 0.909 & 0.920 & 0.992 & 0.988 & 0.971 & 0.915 & 0.833 & 0.966 & 0.953 & 0.620 \\ 364 \mathrm{~nm} \text { Excitation } & 0.691 & 0.803 & 0.934 & 0.570 & -0.46 & -0.32 & -0.22 & 0 & 0 & 0\end{array}$

(b)

\begin{tabular}{lllllllllll} 
Displacement $(\mathrm{mm})$ & 0.25 & 0.75 & 1.25 & 1.75 & 2.25 & 2.75 & 3.25 & 3.75 & 4.25 & 4.75 \\
$485 \mathrm{~nm}$ Excitation & 0.784 & 0.707 & 0.927 & 0.952 & 0.952 & 0.921 & 0.850 & 0.896 & 0.910 & 0.737 \\
$434 \mathrm{~nm}$ Excitation & 0.337 & 0.430 & 0.912 & 0.950 & 0.910 & 0.624 & -0.21 & 0.734 & 0.866 & 0.696 \\
$364 \mathrm{~nm}$ Excitation & -0.05 & 0.108 & 0.757 & 0.658 & 0.460 & 0 & 0 & 0 & 0 & 0 \\
$(\mathbf{c})$ & & & & & & & & & & \\
Displacement (mm) & 0.25 & 0.75 & 1.25 & 1.75 & 2.25 & 2.75 & 3.25 & 3.75 & 4.25 & 4.75 \\
Control & $4.66 e-21$ & $3.14 e-22$ & $1.01 e-47$ & $5.66 e-43$ & $1.86 e-33$ & $9.03 e-22$ & $9.74 e-15$ & $1.35 e-31$ & $3.94 e-28$ & $7.54 e-7$ \\
Diabetic & $2.08 e-2$ & $2.42 e-3$ & $5.18 e-19$ & $5.59 e-24$ & $1.94 e-18$ & $2.78 e-6$ & $1.47 e-1$ & $4.30 e-9$ & $2.89 e-14$ & $5.66 e-8$ \\
\hline
\end{tabular}

First, the lenses of diabetic patients are subject to an accelerated aging process (protein glycation and subsequent Maillard-Amadori reactions). Our experimental data support this hypothesis. While the lenses of control subjects have physiological ages close to their chronological ages, those of a diabetic
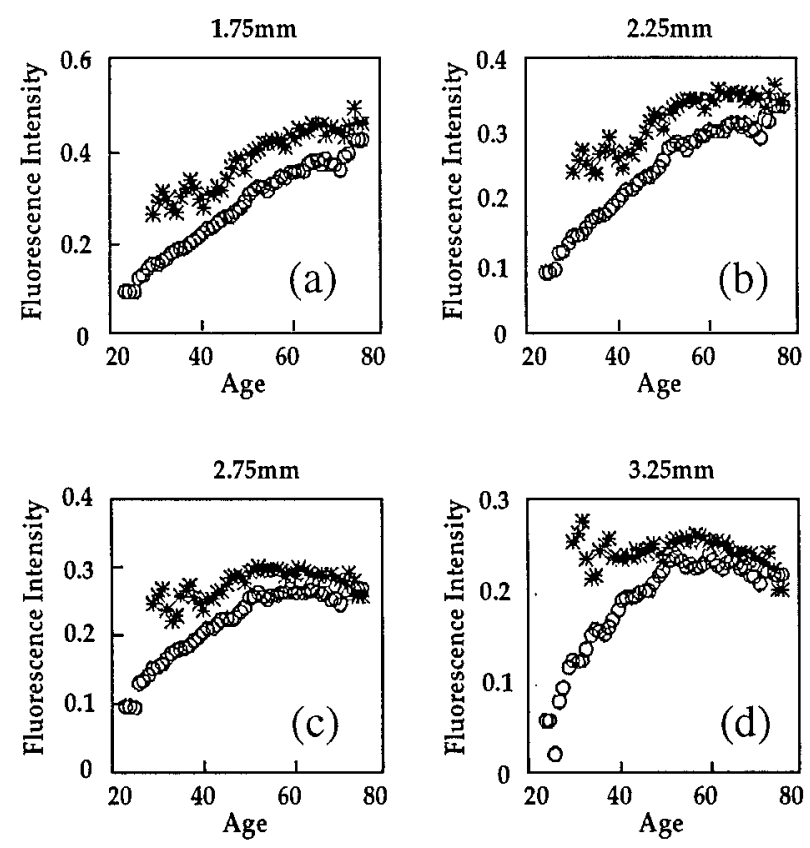

Fig. 4 Relative fluorescence vs. age at (a) 1.75, (b) 2.25, (c) 2.75 , and (d) $3.25 \mathrm{~mm}$ into the lens along the optical axis of controls $(O)$ and diabetic subjects (*). The excitation and collection wavelength $=434 / 495 \mathrm{~nm}$. subject appear to have a physiological age significantly greater than their chronological age. Furthermore, the relationship between physiological and chronological ages in diabetic subjects has a much wider distribution due to the wide variety in severity, duration, and control of diabetes in the subjects.

Second, there may be an attenuation effect due to the absorption of the incident photons before reaching the target and the self-absorption of the emitted photons (fluorescence) on the way out. The actual fluorophore concentrations might continue to increase as subjects' ages increase, yet the in vivo measurement of fluorescence may approach a maximum and perhaps even decline because of the attenuation effect. Therefore, this roll off will affect the linearity of the fluorescence-age relationship. Because it is assumed that diabetic subjects have more fluorescence and therefore more absorption for their chronological age, this roll-off point should be manifested at an earlier age. Also, since absorption effects accumulate exponentially as we scan into the lens, the roll off (hence the difference between controls and diabetic subjects) should become more obvious further into the posterior lens. In comparing the coefficients for the three excitation wavelengths, the correlation appears to be poor at the 364-nm excitation, presumably because of the attenuation effect caused by stronger absorption and possibly scattering.

Figure 4 supports both hypotheses discussed here. The physiological age argument is supported because of the increased spread of diabetes about the fit-line in comparison with the controls. This is most apparent for the younger individuals with 

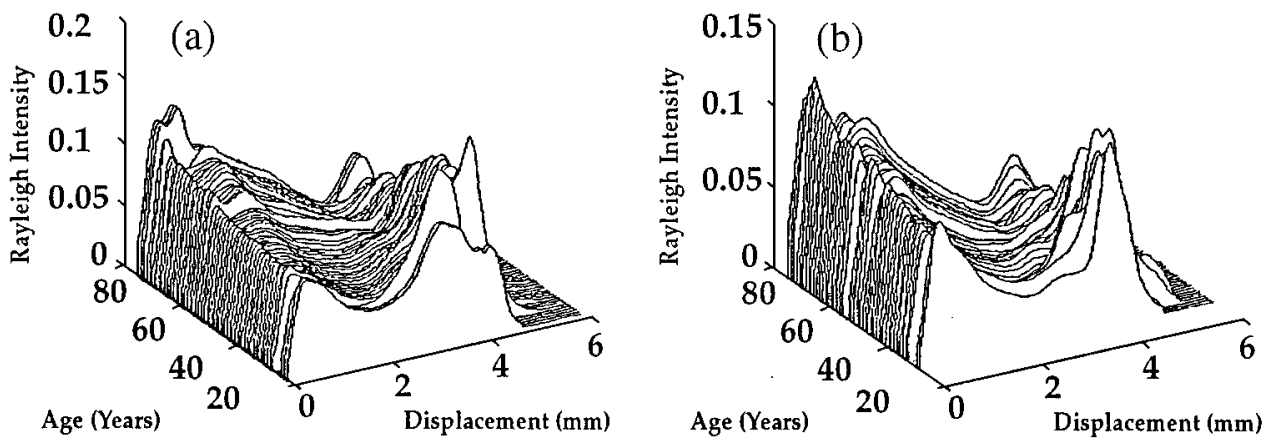

Fig. 5 Relative Rayleigh profiles (or wave forms) for different ages along the optical axis of the lens in (a) controls and (b) diabetic subjects. The excitation (Rayleigh) wavelength is at $434 \mathrm{~nm}$.

diabetes. The absorption theory is more obvious. Figure 4(a) shows an almost linear age relationship for the controls. The diabetic subjects' fluorescence at this measurement distance is starting to roll off at about age 70 . Going $500 \mu \mathrm{m}$ further into the lens [Figure 4(b)] shows a slight nonlinearity in the controls and a further degradation in the diabetic subjects' linearity. The diabetic subjects' roll-off age is decreased from 70 to about 55. Proceeding another $500 \mu \mathrm{m}$ [Figure 4(c)], both populations have obvious nonlinearity. The roll-off points are about 45 and 50 years for diabetic subjects and controls, respectively. Here, absorption is so dominant that it attenuates the measured fluorescence for the older subjects. Figure 4(d), at yet another $500 \mu \mathrm{m}$ deeper into the lens, shows that any increase in the diabetic subjects' fluorescence with age is cancelled out by the attenuation of both the excitation energy and the fluorescence. The control population roll-off point has moved down to about 45 years old.

We have shown that the in vivo measurement of fluorescence in the lens saturates due to absorption effects. Therefore, any population prediction algorithm must take this into account, or the prediction performance will be poor. For example, referring back to Figure 4(a) and 4(c), we see that although there is a clear separation in the two populations for all ages for $4(\mathrm{a})$, at $2 \mathrm{~mm}$ deeper into the lens, the separation is no longer apparent for subjects more than 50 years old.

\subsection{FLUORESCENCE/RAYLEIGH RATIOS}

Earlier preliminary data have already indicated that Rayleigh-normalized fluorescence in the lens provides more separation between the normal and diabetic lens than conventional methods. ${ }^{21}$ The fluorescence/Rayleigh ratios were calculated for the age-binned and averaged profiles we have been examining. The result is a ratio value at each point in the lens (see Figure 7). Let us elaborate as to what effect this Raleigh normalization will have on the fluorescence data with respect to subject population and age as we scan through the lens.

It is readily seen that absorption is inherently embedded in both the measured fluorescence and measured Rayleigh. We may define $F_{a}\left(z, \lambda_{1}, \lambda_{2}\right)$ as the actual fluorescence excited at $\lambda_{1}$, collected at $\lambda_{2}$ at a distance $z$ into the lens:
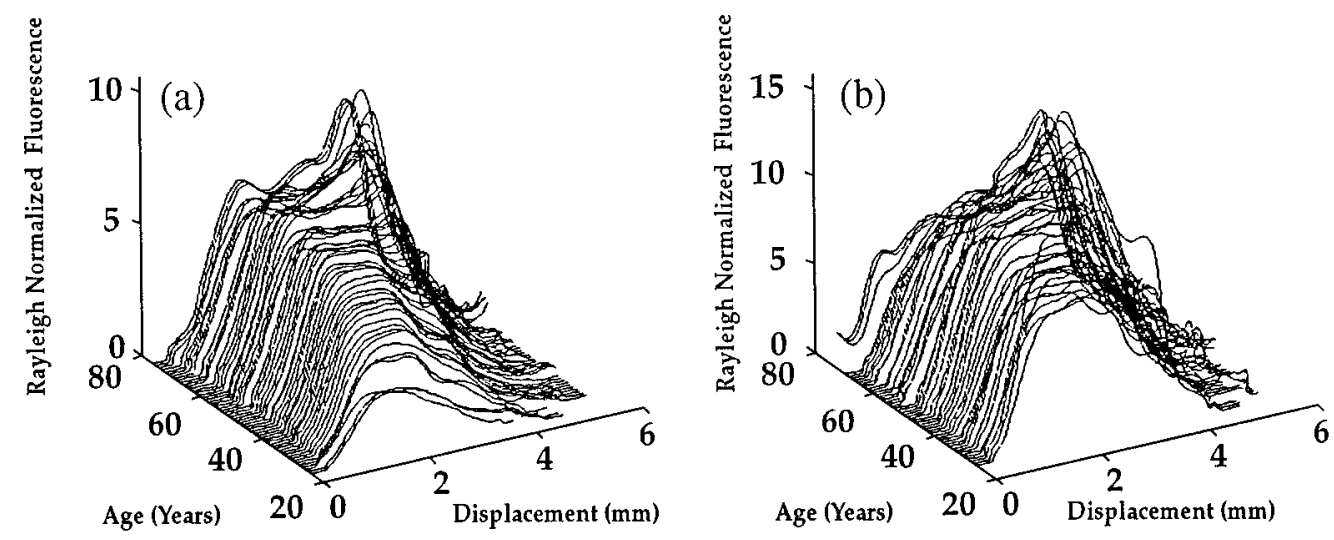

Fig. 6 Rayleigh normalized fluorescence (fluorescence/Rayleigh ratio) for different ages along the optical axis of the lens in (a) controls and (b) diabetic subjects. The excitation and collection wavelength $=434 / 495 \mathrm{~nm}$. 

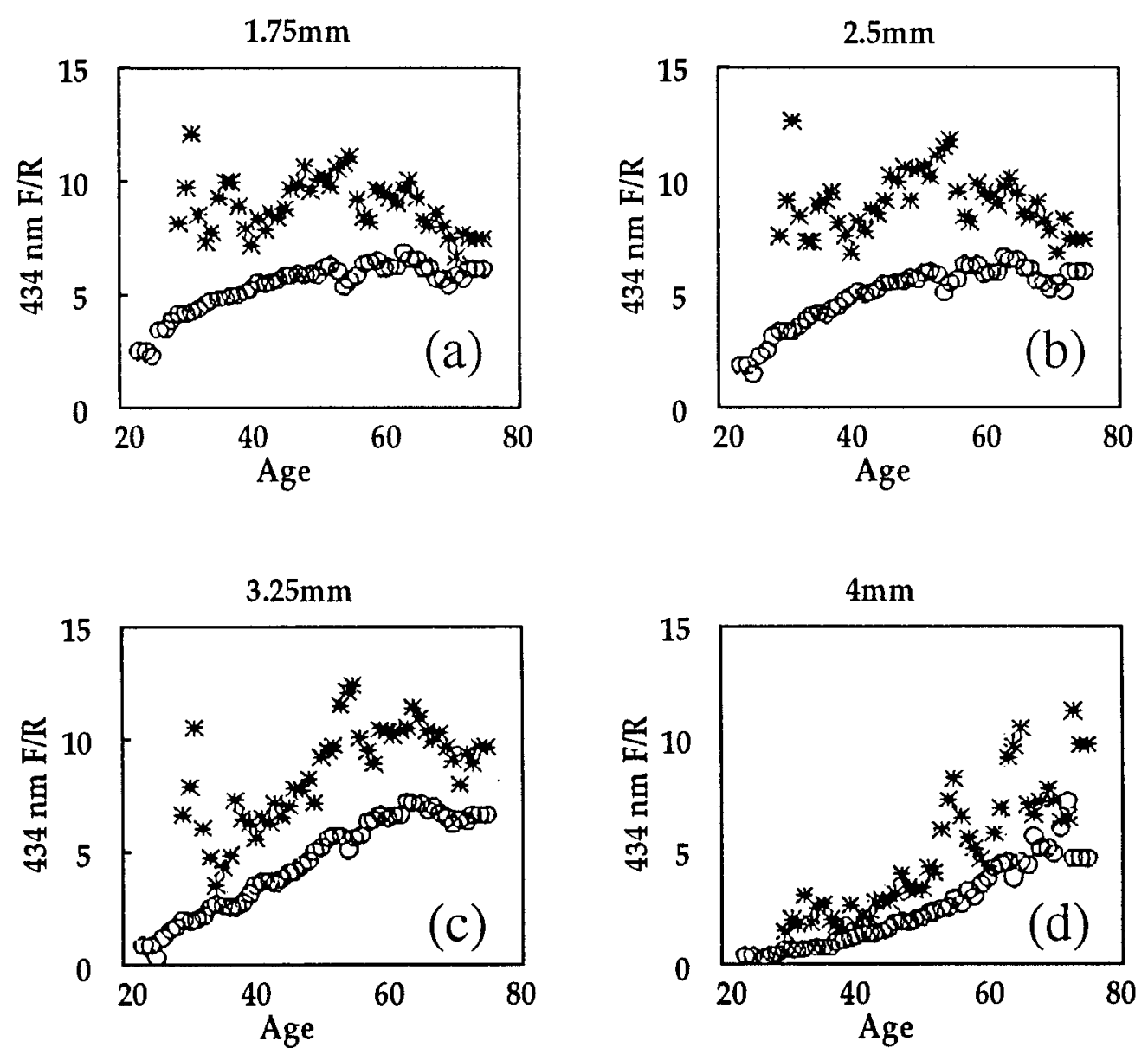

Fig. 7 Rayleigh normalized fluorescence (fluorescence/Rayleigh ratio) vs. age at (a) 1.75, (b) 2.5, (c) 3.25 , and (d) $4 \mathrm{~mm}$ into the lens along the optical axis of controls (O) and diabetic subjects (*). The excitation and collection wavelength $=434 / 495 \mathrm{~nm}$.

$$
\begin{aligned}
F_{a}\left(z, \lambda_{1}, \lambda_{2}\right)= & F_{m}\left(z, \lambda_{1}, \lambda_{2}\right) \exp \left[\int _ { 0 } ^ { z } \left\{\alpha_{\lambda_{1}}(w)\right.\right. \\
& \left.\left.+\alpha_{\lambda_{2}}(w)\right\} d w\right]
\end{aligned}
$$

where $F_{m}$ is the measured fluorescence, and $\alpha_{\lambda}(z)$ is the absorption profile of the lens, which is $\lambda$-dependent. Likewise, we may define $R_{a}\left(z, \lambda_{1}\right)$ as the actual Rayleigh at $\lambda_{1}$ and $z$ :

$$
R_{a}\left(z, \lambda_{1}\right)=R_{m}\left(z, \lambda_{1}\right) \exp \left[2 \int_{0}^{z} \alpha_{\lambda_{1}}(w) d w\right] .
$$

In fact, $R_{a}$ depends on the angle of scattering. However, because of the pupil tracker in our LMS, the angle remains fixed for all measurements and patients. The 2 in the exponent in Eq. (2) is due to the absorption of the excitation beam and the collection beam. Note that in Eq. (1), if $\lambda_{1}=\lambda_{2}$, one would also get a 2 in the exponent. From the ratio of Eqs. (1) and (2) and upon rearrangement, one obtains the following expression for calculating the ratio of measured values:

$$
\begin{aligned}
\frac{F_{m}\left(z, \lambda_{1}, \lambda_{2}\right)}{R_{m}\left(z, \lambda_{1}\right)}= & \frac{F_{a}\left(z, \lambda_{1}, \lambda_{2}\right)}{R_{a}\left(z, \lambda_{1}\right)} \exp \left[\int _ { 0 } ^ { z } \left\{\alpha_{\lambda_{1}}(w)\right.\right. \\
& \left.\left.-\alpha_{\lambda_{2}}(w)\right\} d w\right] .
\end{aligned}
$$

We see that the absorption contribution is almost corrected for, leaving only the difference in absorption at the two wavelengths. It should be noted that since $\alpha_{\lambda_{2}}$ will always be less than $\alpha_{\lambda_{1}}$ in the human lens in the 360 to $600-\mathrm{nm}$ region, ${ }^{22}$ the absorption factor will always be positive. Furthermore, as we measure deeper into the lens, the absorption effects increase and consequently so does the ratio of fluorescence to Rayleigh. It is of interest to see if the experimental data agree with the above theory.

Two key observations can be made from Figure $7(\mathrm{a})$ to $7(\mathrm{~d})$. First, the theory presented above is supported. At the more shallow depths into the lens, the ratio values for both the control and diabetic subjects roll off with age, as did the fluorescence values in Figure 4(a) to 4(d). However, as we scan deeper into the lens, we can see that the roll- 

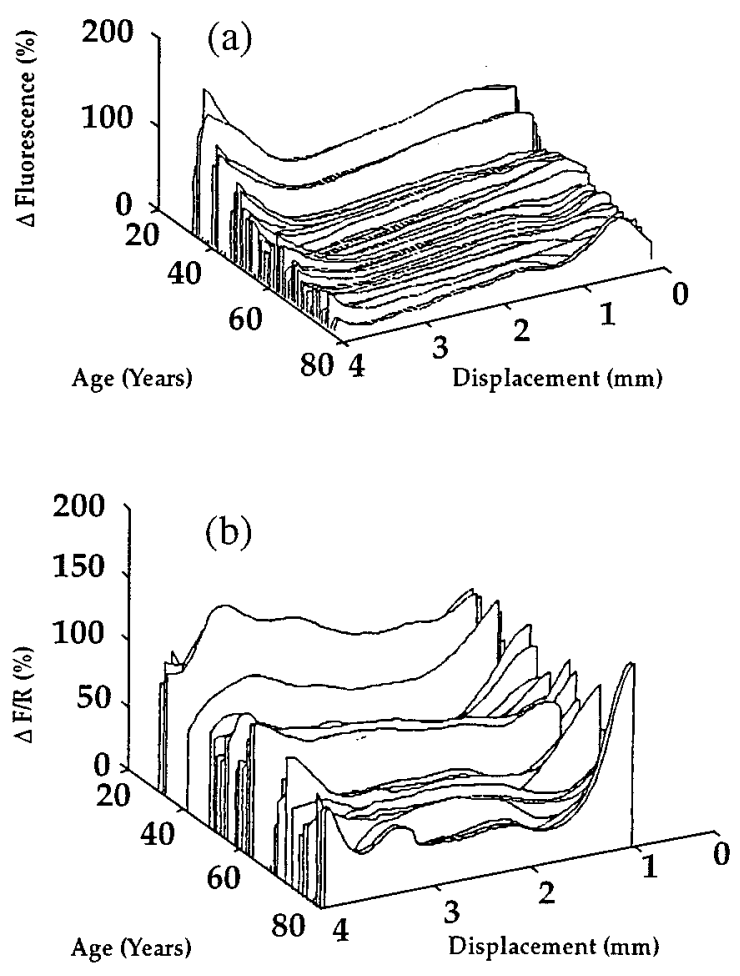

Fig. 8 (a) Percent difference in the fluorescence of the two populations vs. age and displacement; (b) percent difference in the Rayleigh normalized fluorescence of the two populations vs. age and displacement. off point does not decrease as it did with only fluorescence intensity. On the contrary, the roll off becomes less obvious. In fact, at a depth of $4 \mathrm{~mm}$ [Figure $7(\mathrm{~d})$ ], it appears that the ratio values increase in an exponential relationship with age. This agrees with the equations above: the deeper we go, the more of an effect absorption has. As absorption effects increase, the ratio values increase as well.

The second observation can be made by contrasting Figure 4(a) and 4(d) with Figure 7(a) and 7(c). These plots are at comparable distances into the lens $(1.75$ and $3.25 \mathrm{~mm})$. We showed that in Figure $4(d)$, absorption effects had become so dominant that the fluorescence differences between the two populations was removed in the older patients. However, Figure 7(c) does not show this degradation in separation. To discuss the data quantitatively, refer to Figure 8(a) and 8(b). These are the percent differences between the fluorescence [Figure 8(a)] and Rayleigh normalized fluorescence [Figure 8(b)] versus age and displacement. Figure $8(\mathrm{a})$ and 8 (b) can be summarized by Tables 2(a) to 2(c). Table 2(a) shows the difference in the fluorescence of the two populations; Table 2(b) shows the difference in the Rayleigh normalized fluorescence of the two populations; and Table 2(c) shows the difference between the values in Tables 2(b) and 2(a) (Rayleigh normalized difference-fluorescence difference). By examining Table 2(c), one sees that

Table 2 (a) Differences between diabetes and control fluorescence (\%). (b) Differences between diabetes and control Rayleigh normalized fluorescence (\%). (c) Improvement in population separation between Rayleigh normalized fluorescence and fluorescence (2b-2a).

\begin{tabular}{|c|c|c|c|c|c|c|c|c|c|}
\hline & & $Z=0.75 \mathrm{~mm}$ & 1.25 & 1.75 & 2.25 & 2.75 & 3.25 & 3.75 & 4.25 \\
\hline \multicolumn{10}{|c|}{ (a) } \\
\hline Age & 30 & 78 & 66 & 59 & 54 & 50 & 71 & 108 & -200 \\
\hline & 40 & 25 & 23 & 21 & 18 & 15 & 21 & 32 & 32 \\
\hline & 50 & 34 & 30 & 24 & 17 & 14 & 6 & 19 & 56 \\
\hline & 60 & 30 & 21 & 18 & 13 & 9 & 6 & 9 & 28 \\
\hline & 70 & 21 & 23 & 21 & 17 & 13 & 11 & 16 & 22 \\
\hline \multicolumn{10}{|c|}{ (b) } \\
\hline Age & 30 & 114 & 88 & 82 & 89 & 97 & 117 & 104 & 101 \\
\hline & 40 & 75 & 41 & 31 & 31 & 34 & 42 & 41 & 31 \\
\hline & 50 & 81 & 59 & 53 & 60 & 62 & 59 & 52 & 39 \\
\hline & 60 & 78 & 43 & 43 & 48 & 47 & 47 & 16 & 15 \\
\hline & 70 & 48 & 26 & 32 & 39 & 41 & 38 & 50 & 29 \\
\hline \multicolumn{10}{|c|}{ (c) } \\
\hline Age & 30 & 36 & 22 & 23 & 35 & 47 & 46 & -4 & 301 \\
\hline & 40 & 50 & 18 & 10 & 13 & 19 & 21 & 9 & -1 \\
\hline & 50 & 47 & 29 & 29 & 43 & 48 & 53 & 33 & -17 \\
\hline & 60 & 48 & 22 & 25 & 35 & 38 & 41 & 7 & -13 \\
\hline & 70 & 27 & 3 & 11 & 22 & 28 & 27 & 34 & 7 \\
\hline
\end{tabular}


using Rayleigh profiles to normalize fluorescence compensates for absorption and greatly improves the overall separations between diabetic subjects and controls.

In summary, we have shown that the measured fluorescence in the lens saturates due to absorption effects. Therefore, any population prediction algorithm must take this into account, or the prediction performance will be poor. We have also shown that by using the measured Rayleigh profiles to normalize the measured fluorescence, most of the absorption effects are removed. When discussing the separation between the fluorescence of diabetic subjects and controls, this Rayleigh normalization creates a definite improvement. This improvement consists of decreasing the age dependency of the separation, as well as increasing the overall magnitude of the separation.

\section{REFERENCES}

1. R. van Heyningen, "Fluorescence glucoside in the human lens," Nature (London) 203, 393-394 (1971).

2. N.-T. Yu, B. C. Barron, and J. F. R. Kuck, Jr., “Distribution of two metabolically related fluorophores in human lens measured by laser microprobe," Exp. Eye Res. 49, 189-194 (1989).

3. N.-T. Yu, M.-Z. Cai, D. J.-Y. Ho, and J. F. R. Kuck, Jr., "Automated laser-scanning-microbeam fluorescence/Raman image analysis of human lens with multichannel detection: evidence for metabolic production of a green fluorophore," Proc. Natl. Acad. Sci. U.S.A. 85, 103-106 (1988).

4. S. Zigman, "Eye lens color: formation and function," Science 171, 807-809 (1971).

5. R. F. Borkman, "Ultraviolet spectrum for tryptophan destruction in aqueous solution," Photochem. Photobiol. 26, 163166 (1977).

6. J. S. Zigler, Jr. and J. D. Goosey, "Photosensitized oxidation in the ocular lens: evidence for photosensitizers endogenous to the human lens," Photochem. Photobiol. 33, 869-874 (1981).

7. A. Pirie, "Formation of $N$-formyl kynurenine in proteins from lens and other sources by exposure to sunlight," Biochem. J. 125, 203-207 (1971).

8. J. Dillon, A. Spector, and K. Nakamishi, "Identification of $\beta$-carbolines isolated from fluorescent lens proteins," Nature 254, 422-423 (1976).

9. R. J. W. Truscott, K. Faull, and R. C. Augusteyn, "The identification of anthranilic acid in proteolytic digests of cataractous lens proteins," Ophthalmic Res. 9, 263-267 (1977).

10. S. Garcia-Castineiras, J. Dillon, and A. Spector, "Nontryptophan fluorescence associated with human lens proteins: apparent complexity and isolation of bityrosine and anthranilic acid," Exp. Eye Res. 26, 461-467 (1978).

11. V. M. Monnier and A. Cerami, "Detection of non-enzymatic browning products in human lens," Biochim. Biophys. Acta. 760, 97-103 (1983).

12. J. Nordmann, "Problems in cataract research," Ophthalmic Res. 3, 323-359 (1972).

13. G. J. H. Bessems, E. Keizer, J. Wollensak, and H. J. Hoenders, "Non-tryptophan fluorescence of crystallins from normal and cataractous human lenses," Invest. Ophthalmol Vis. Sci. 28, 1157-1163 (1987).

14. M. Bando, A. Nakajima, and K. Satoh, "Spectrophotometric estimation of 3-OH-L-kynurenine-O- $\beta$-glucoside in the human lens," J. Biochem. 89, 103-107 (1981).

15. N.-T. Yu, M. Bando, and J. F. R. Kuck, Jr., "Fluorescence/ Raman intensity ratio for monitoring the pathologic state of human lens," Invest. Ophthalmol. Vis. Sci. 26, 97-101 (1985).

16. N.-T. Yu, J. F. R. Kuck, Jr., and C. C. Askren, "Red fluorescence in older and brunescent human lenses," Invest. Ophthalmol. Vis. Sci. 18, 1278-1280 (1979).

17. V. M. Monnier and A. Cerami, "Detection of non-enzymatic browning products in human lens," Biochim. Biophys. Acta. 760, 97-103 (1983).

18. V. M. Monnier and A. Cerami, "Nonenzymic glycosylation and browning of proteins in diabetes," Clin. Endocrinol. Metab. 11 (2), 431-452 (1982).

19. F. G. Njoroge and V. M. Monnier, "The chemistry of the Maillard reactions under physiological conditions: a review," Prog. Clin. Biol. Res. 304, 85-107 (1988).

20. J. W. Baynes, N. G. Watkins, C. I. Fisher, C. J. Hull, J. S. Patrick, M. U. Ahmed, J. A. Dunn, and S. R. Thorpe, "The Amadori product on protein: structure and reactions," Prog. Clin. Biol. Res. 304, 43-67 (1988).

21. M. A. Samuels, S. W. Patterson, J. A. Eppstein, N.-T. Yu, and S.-E. Bursell, "Apparatus and methods for quantitatively measuring molecular changes in the ocular lens," U.S. Patent No. 5,203,328 (1993).

22. S. Lerman, Radiant Energy and the Eye, Macmillan, New York (1980). 